\title{
Soliton molecules with two frequencies
}

\author{
Oliver Melchert, ${ }^{1,2,3}$ Stephanie Willms, ${ }^{1,2}$ Surajit Bose ${ }^{2}$ Alexey Yulin, ${ }^{4}$ Bernhard Roth, ${ }^{1,3}$ \\ Fedor Mitschke, ${ }^{5}$ Uwe Morgner, ${ }^{1,2,3}$ Ihar Babushkin, ${ }^{1,2}$ and Ayhan Demircan ${ }^{1,2,3}$ \\ ${ }^{1}$ Cluster of Excellence PhoenixD, Welfengarten 1, 3016\%, Hannover, Germany \\ ${ }^{2}$ Institute of Quantum Optics, Leibniz Universität Hannover, Welfengarten 1, 30167, Hannover, Germany \\ ${ }^{3}$ Hannover Centre for Optical Technologies, Nienburger Str. 17, 30167, Hannover, Germany \\ ${ }^{4}$ Department of Nanophotonics and Metamaterials, ITMO University, \\ Birzhevaya Liniya V.O., 14, 199034, Saint Petersburg, Russia \\ ${ }^{5}$ Institute for Physics, University of Rostock, 18059 Rostock, Germany
}

(Dated: November 14, 2019)

\begin{abstract}
We demonstrate a peculiar mechanism for the formation of bound states of light pulses of substantially different optical frequencies, in which pulses are strongly bound across a vast frequency gap. This is enabled by a propagation constant with two separate regions of anomalous dispersion. The resulting soliton compound exhibits molecule-like binding energy, vibration, and radiation and can be understood as a mutual trapping providing a striking analogy to quantum mechanics. The phenomenon constitutes an intriguing case of two light waves mutually affecting and controlling each other.
\end{abstract}

Solitons are ubiquitous in nature and play an important role in as diverse fields of science as fundamental physics, mathematics, and applications in optical communications [1 [5]. They are important to understand the physics of Bose-Einstein condensates [6], certain biological systems [7, and more. The most intriguing property of solitons is their particle-like propagation as localized energy packages, held together by a balance between linear diffraction or dispersion and nonlinear effects. This particular wave-particle dualism allows the investigation of phenomena which are usually attributed to quantum mechanical principles, insofar as they can be described by nonlinear theories. The elastic particlelike collision of waves 3 is an example. In direct extension of this concept of quantum analogy, the phenomenon of molecule-like soliton states $[\underline{8}$ can be treated in terms of binding energy or vibration, and deeper insight into complex soliton interaction processes like collisions [9], trapping [10], or the pervasive rogue wave phenomenon [1] can be obtained. At the same time soliton molecules are of tremendous interest for application in optical technologies, because they may provide alternative coding schemes for transmitting information with enhanced data-carrying capacity [12. As diverse as the contexts in which solitons appear are the possible realizations of soliton molecules. Usually soliton molecules are associated with double-hump intensity profiles, consisting of two solitons at the same center frequency, copropagating unchanged together as a bound state. Due to a certain phase relationship between the two pulses, a balance of attractive and repulsive forces between the constituents of the pulse doublet is established [13]. In their temporal versions, they appear in nonlinear optical fibers governed by the generalized nonlinear Schrödinger equation (NSE) [14, the dissipatively perturbed NSE [15], coupled NSEs describing twin-core fibers [16, or the complex Ginzburg-Landau equation [17]. There are also numerous realizations of further soliton molecules [18 27.

We present here a previously unreported class of temporal soliton molecules. They consist of two pulses at widely different frequencies which, however, are bound by Kerr forces. In contrast to this double-peaked spectral profile, the temporal shape of the compound state is close to that of an individual soliton, except that it

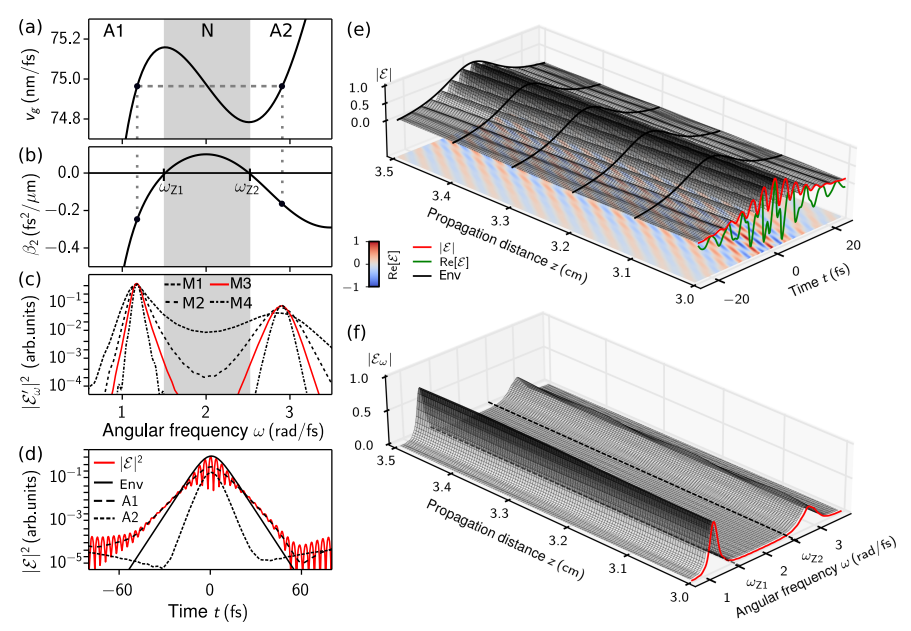

FIG. 1. Two frequency molecule boundstates. (a) Groupvelocity-, (b) group-velocity-dispersion profile with anomalous dispersion regimes $\mathrm{A} 1, \mathrm{~A} 2$ and normal dispersion regime $\mathrm{N}$ (shaded in grey). Dots identify loci of selected groupvelocity matched solitons. (c) Output spectra of selected molecules M1-M4 for different initial pulse widths. (d) Temporal intensity profile $|\mathcal{E}|^{2}$ (M3). Dashed and dotted lines show components in $\mathrm{A} 1$ and $\mathrm{A} 2$, respectively. Solid black line: adjusted hyperbolic secant governing envelope. (e) Temporal evolution (M3) (indicated by a red line). Gray surface denotes absolute value of $\mathcal{E}$, green line the electric field. Its evolution as contour plot is projected onto the $(t, z)$-plane, and (f) spectral evolution. 
is dressed by interference fringes. Key to understanding these novel structures is a strong attractive potential between two solitons: each soliton is trapped by a potential well created by another soliton. This leads to a strong binding energy and robustness against perturbations. This effect is an inversion of the strong repulsive potential between a quasi co-propagating soliton and dispersive wave [28, 29].

As we aim to consider widely separated frequency components, the standard nonlinear Schrödinger equation is insufficient because it uses the concept of a center frequency. Therefore we use a non-envelope variant,

$$
i \partial_{z} \mathcal{E}_{\omega}+\left[\beta(\omega)-\omega / v_{0}\right] \mathcal{E}_{\omega}+\frac{3 \omega^{2} \chi}{8 c^{2} \beta(\omega)}\left(|\mathcal{E}|^{2} \mathcal{E}\right)_{\omega>0}=0
$$

suited for unidirectional propagation in terms of the analytic signal $\mathcal{E}(z, t)=2 \int_{\omega>0} \mathrm{~d} \omega E_{\omega}(z) e^{-i \omega t}[30$. $\chi$ refers to the Kerr nonlinearity, $c$ to the speed of light, and $\beta(\omega)$ to the propagation constant. This equation includes the evolution of the optical field $E(z, t)=\int \mathrm{d} \omega E_{\omega}(z) e^{-i \omega t}$ during single mode propagation over distance $z$ in a nonlinear waveguide. A modified linear coefficient function $\beta(\omega)-\omega / v_{0}$ is introduced so that we directly obtain the dynamics in a reference frame moving with velocity $v_{0}$. The main prerequisite for our molecule states is group-velocity matched co-propagation of pulses at different frequencies in anomalous dispersion regimes. This can be realized in waveguides with a dispersion profile exhibiting two anomalous dispersion regimes separated by a normal one, as given, e.g., in microstructured fibers 33, silicon slot waveguides [34, 35, or Kagome-fibers allowing for several adjustable separate anomalous dispersion regimes [36. A paradigmatic profile of the frequency dependent group velocity $v_{g}$ and dispersion profile $\beta_{2}$ are shown in Figs. 1(a,b). Such a system has already been shown to exhibit peculiar dynamics, resembling quantum mechanical behavior. Soliton spectral tunneling between phase-matched anomalous dispersion regimes has been shown [20, 31. We will demonstrate that the system exhibits further intriguing analogies to a quantum mechanical systems. This is the case when dynamics are not determined by phase relations. The main underlying mechanism is then similar to the wave reflection phenomenon originating from fluid dynamics 37 and known in optics as the optical push broom effect 38, temporal reflection 39, or as the concept of an optical event horizon [40], facilitating strong and highly efficient all-optical interaction [28. However, in contrast to these, interaction between pulses in separated regimes of anomalous dispersion is attractive as we will demonstrate below. To use this strong force we inject as a first example the superposition of two fundamental solitons $\sqrt{\chi} E(0, t)=$ $\operatorname{Re}\left[\mathcal{A}_{1} e^{-i \omega_{1} t} / \cosh \left(t / t_{1}\right)+\mathcal{A}_{2} e^{-i \omega_{2} t} / \cosh \left[(t-\delta) / t_{2}\right]\right]$ with amplitudes $\mathcal{A}_{1 / 2}$, at center frequencies $\omega_{1 / 2}=1.2 / 2.939 \mathrm{rad} /$ fs (black dots in Figs. 1 $(\mathrm{a}, \mathrm{b})$ ), and
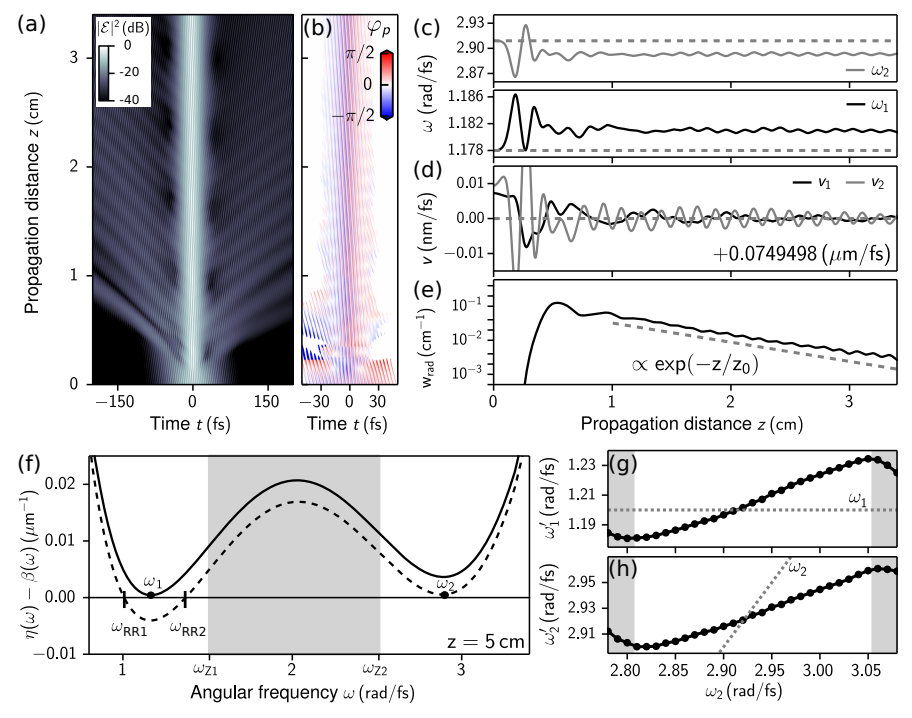

FIG. 2. Radiating molecule states. (a) Evolution of $|\mathcal{E}|^{2}$ (a movie is provided in the supplemental material [42]). (b) Corresponding instantaneous phase $\varphi_{p}$. (c) Evolution of the center frequencies of the molecule's frequency components $\omega_{1 / 2}$. (d) Velocities $v_{1 / 2}$ of the sub-pulse centroid. (e) Emitted photon energy rate $w_{\text {rad }}$ (see [4]). (f) Phase-matching analysis for both subpulses yielding two resonant frequencies $\omega_{\text {RR1/RR2 }}$. (g) Parameter study indicating the drift of the asymptotic sub-pulse center frequency $\omega_{1}^{\prime}$ relative to its initial value $\omega_{1}$ (dashed line) upon variation of $\omega_{2}$. (h) Same for $\omega_{2}^{\prime}$. Shaded areas mark regions where one soliton dominates the dynamics.

at delay $\delta=0$ fs into the waveguide. We have deliberately chosen an asymmetric dispersion profile to increase the manifold of possible compounds due to unequal soliton combinations. More importantly, the anomalous dispersion regions are vastly separated so as to avoid any initial spectral overlap, which clearly distinguishes our approach from others [19, 41]. As initial conditions for the soliton parameters we set $t_{1 / 2}=20 \mathrm{fs}$, and $\mathcal{A}_{1 / 2}$ corresponding to fundamental solitons (see supplemental material 42]). Figure 1(e) represents the asymptotic state well after the initial transient phase. The electric field is shown as a green curve, and its evolution as a contour plot is projected on the $(t, z)$-plane. In the temporal domain the molecule state is a single pulse with deep interference fringes that propagates without shape variation. With $\Delta \omega=\omega_{2}-\omega_{1} \approx 1.74 \mathrm{rad} / \mathrm{fs}_{\mathrm{s}}$ as stated above, $|\mathcal{E}(z, t)|^{2} \propto \cos ^{2}(\Delta \omega t / 2)$ so that the fringe period is $\Delta t \approx 3.61 \mathrm{fs}$.

It is remarkable that the governing envelope of this pulse is of nearly perfect hyperbolic secant shape, just like a fundamental soliton, as is highlighted by the black solid lines in Figs. 1 $(\mathrm{d}, \mathrm{e})$. This can be described by a meta-envelope [43, introduced to describe the properties of a general class of solitons in a system similar to ours. An important point here is that the energy content of the molecule is lower than the sum of the initial 
solitons. The asymptotic fraction of energy localised in the molecule-state does not exceed $92 \%$, which makes it clear that the solution described here is profoundly different from plain co-propagation of two fundamental solitons. In the spectral domain the molecule has a peak in each of the separated regions of anomalous dispersion; these two peaks are no longer centered on perfectly group-velocity matched frequencies, but undergo small oscillations around the group-velocity of the compound state.

Stable molecules can be created over a wide range of initial soliton parameters, with a spectrum consisting either of well separated parts in the two anomalous dispersion regimes or with overlapping parts extending over the interjacent normal dispersion regime. We performed a series of simulations changing the temporal width, but always starting with amplitudes for fundamental solitons at the corresponding frequencies. Selected examples of the spectra with increasing energy content are shown in Fig. 1(c). Holding an extended fraction of spectral energy in the region of normal dispersion, the governing envelope severely deviates from a perfect hyperbolic secant. To investigate the robustness of the molecules and elucidate a further analogy to the quantum mechanical molecules, we now change the frequency relation of the initial solitons with increasing group-velocity mismatch. An example is shown in Fig. 2(a), demonstrating the propagation of a radiating molecule. The strong oscillations at small distances $z$ involve strong emission of radiation dependent on the initial group-velocity mismatch. The instantaneous phase in the vicinity of the molecule state is illustrated in Fig. 2(b) (see supplemental material 42]), showing a regular evolution pattern without strong perturbations. The evolution of the newly adapted $\omega$-centroids in the two separated anomalous dispersion regimes are depicted in Fig. 2(c), with dashed lines corresponding to initial center frequencies. The velocities of the pulse $t$ centroids are provided in Fig. 2(d). These quantities are subject to vigorous variations during the initial transient phase of molecule formation. The loss of energy due to radiation is shown in Fig. 2(e), exhibiting an exponential decay $\propto \exp \left(-z / z_{0}\right)$ with $z_{0}=0.77 \mathrm{~cm}$ (indicated by the dashed line). This behavior shows similarities to molecular vibration and the generation of dipole-like radiation. Mismatch within certain limits still enables formation of molecules which compensate its destabilizing effect through frequency shifts. The interaction between parts located in different anomalous dispersion regions and generation of radiation is highly complex due to the mutually induced refractive index changes, yet can in part be understood by phase-matching conditions. In this regard we transferred the theoretical approach detailed in Refs. 16, 32, 44, to the present case.

We separated the molecule into two sub-pulses without spectral overlap by filtering in the frequency domain. Following Ref. 32] we determined the phase-matching
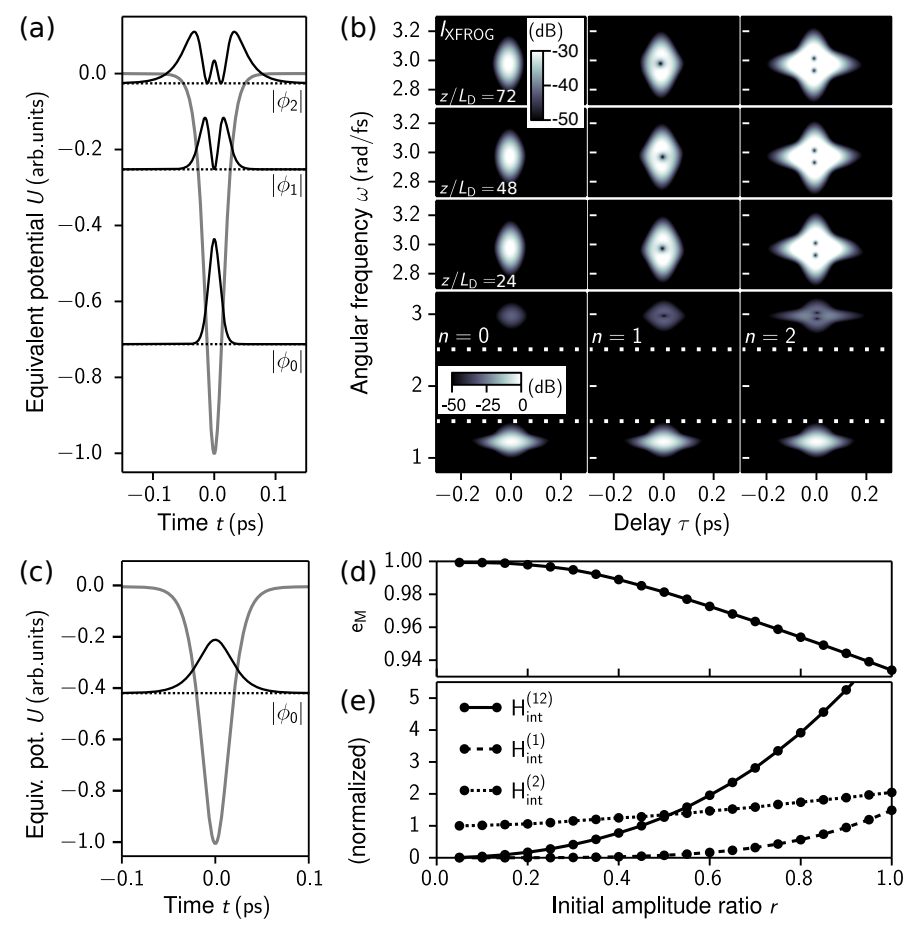

FIG. 3. Trapped states in a propagating refractive index well. (a) Effective potential with three eigenstates $\phi_{0}, \phi_{1}$, and $\phi_{2}$, indicated at a depth corresponding to their eigenvalues (horizontal dotted lines). (b) From bottom to top: XFROG-traces of initial configurations of the soliton and its weak eigenstates (labeled $n=0,1,2)$. Horizontal dotted lines indicate the zero-dispersion frequencies. Close up views of eigenstates at selected propagation distances in multiples of the dispersion length $L_{D}=t_{s}^{2} /\left|\beta_{2}\left(\omega_{s}\right)\right|$. (c) Potential with a single eigenstate. (d) Fractional contribution $\mathrm{e}_{M}$ of the compound pulse to the total energy (at $z=0.3 \mathrm{~m}$ ). (e) Normalized values of self- $\mathrm{H}_{\mathrm{int}}^{(1)}, \mathrm{H}_{\mathrm{int}}^{(2)}$, and mutual interaction $\mathrm{H}_{\mathrm{int}}^{(12)}$.

resonance frequencies $\omega_{\mathrm{RR}}$ by finding the roots $\eta\left(\omega_{\mathrm{RR}}\right)-$ $\beta\left(\omega_{\mathrm{RR}}\right)=0$, with $\eta(\omega)=\gamma P_{0} / 2+\beta\left(\omega_{0}\right)+\beta_{1}\left(\omega_{0}\right)\left(\omega-\omega_{0}\right)$, for both molecule subpulses. The values of $P_{0}$ and $\omega_{0}$ were obtained by separately characterizing each subpulse (see supplemental material for details). We find that radiation is generated at two frequencies $\omega_{\mathrm{RR} 1}$ and $\omega_{\mathrm{RR} 2}$ phase-matched to the sub-pulse in A2 (Fig. 2(f)), both lying in the anomalous dispersion regime $\mathrm{A} 1$ (in this case there are no such resonant frequencies in $\mathrm{A} 2$ or $\mathrm{N}$ ). The possibility of transfer of energy from one anomalous dispersion regime to the other due to phase-matching and the dependence on third- and higher-order dispersion has been investigated in details in connection with the soliton spectral tunnelling phenomenon [32. Here, the mechanism leads to dipole-like radiation. The excitation depends on the periodic evolution of the peak intensity of the centroid frequency $\omega_{2}$, cf. Fig. 2(c). Even for initial group-velocity mismatches, the two centroid frequencies can evolve to form a mutually bound state. This is demonstrated in Figs. 2 (g,h) by sweeping $\omega_{2}$ over a range 
of initial frequencies while keeping $\omega_{1}$ fixed. The radiation vanishes asymptotically and plays a minor role for the energy balance of the compound state, which is dominated by the mutual attraction between the sub-pulses at the centroid frequencies.

It is an important point that the frequencies of the two sub-pulses in the molecule are not phase-matched to each other and energy interchange relies on more global conservation laws obeyed by Eq. (1). In the following we will demonstrate a further peculiarity: the molecule state can be understood as mutually trapped states and the conservation laws define a binding energy.

For this purpose, we first regard a weak localized field pulse trapped within a refractive index well created by a soliton (see supplemental material for the derivation and more details). The weak pulse and the soliton are located in separate anomalous dispersion regimes. The group-velocity matching condition allows us to regard a linearised auxiliary problem for the weak pulse. Our derivation leads to a Schrödinger-type eigenvalue equation $\left(d^{2} / d t^{2}+2 \kappa_{n} /\left|\beta_{2}\right|-U(t)\right) \phi_{n}(t)=$ 0 , for the bound eigenstates $\phi_{n}(t)$ (wavenumber $\kappa_{n}<$ $0)$. These correspond to trapped states of the crossphase modulation induced effective potential $U(t)=$ $-4\left(\gamma / \gamma_{s}\right)\left(\left|\beta_{2, s}\right| /\left|\beta_{2}\right|\right) \operatorname{sech}^{2}\left(t / t_{s}\right) / t_{s}^{2}$, wherein $\beta_{2, s}$ and $\gamma_{s}$ are taken at the soliton center frequency $\omega_{s}$ (see gray line in Figs. 3(a,c)). The potential $U(t)$ results from the refractive index well of a co-propagating soliton $s$. The eigenvalue problem can be solved exactly (the respective eigenvalues and eigenfunctions specified by the Gaussian hypergeometric function are listed in the supplemental material). Examples of eigenfunctions lying in the anomalous dispersion regime A2 for a potential created by a soliton with center frequency $\omega_{s}=1.2319 \mathrm{rad} / \mathrm{fs}$ and temporal duration $t_{s}=20 \mathrm{fs}$ are depicted in Fig. 3(a), exhibiting three eigenstates. In our system the number of eigenstates is solely controlled by the center frequencies of both pulses. Numerical simulations summarized in Fig. 3(b) directly verify that Eq. (1) supports analytically calculated trapped states by a potential well created by a soliton.

To come closer to a molecule state, we regard now an associated effective potential with only a single bound-state (Fig. 3(c)) by tuning the center frequency $\omega_{s}$ and monitor the change of the solution by increasing the energy of the bound state. An important value which entails the degree of mutual interaction between the two sub-pulses is given by the interaction term of the conserved momentum flux [30] $\mathrm{H}_{\text {int }}[\mathcal{B}]=\frac{3}{8} \sum_{1 \overline{2} 3 \overline{4} \mid} T_{1234} \mathcal{B}_{\omega_{1}} \mathcal{B}_{\omega_{2}}^{*} \mathcal{B}_{\omega_{3}} \mathcal{B}_{\omega_{4}}^{*}$, where the canonical variable $\mathcal{B}_{\omega}=\sqrt{|\beta(\omega)| /\left(2 \mu_{0} \omega^{2}\right)} \mathcal{E}_{\omega}$, and $T_{1234}=\mu_{0} \chi\left|\omega_{1} \omega_{2} \omega_{3} \omega_{4}\right| / c^{2} \sqrt{\left|\beta\left(\omega_{1}\right) \beta\left(\omega_{2}\right) \beta\left(\omega_{3}\right) \beta\left(\omega_{4}\right)\right|}$. The sum-index token $1 \overline{2} 3 \overline{4} \mid$ abbreviates the condition $\omega_{1}-\omega_{2}+\omega_{3}-\omega_{4}=0$. From this, the self-interaction parts of the pulses $\mathrm{H}_{\mathrm{int}}^{(1)}$ and $\mathrm{H}_{\mathrm{int}}^{(2)}$, as well as their mu-
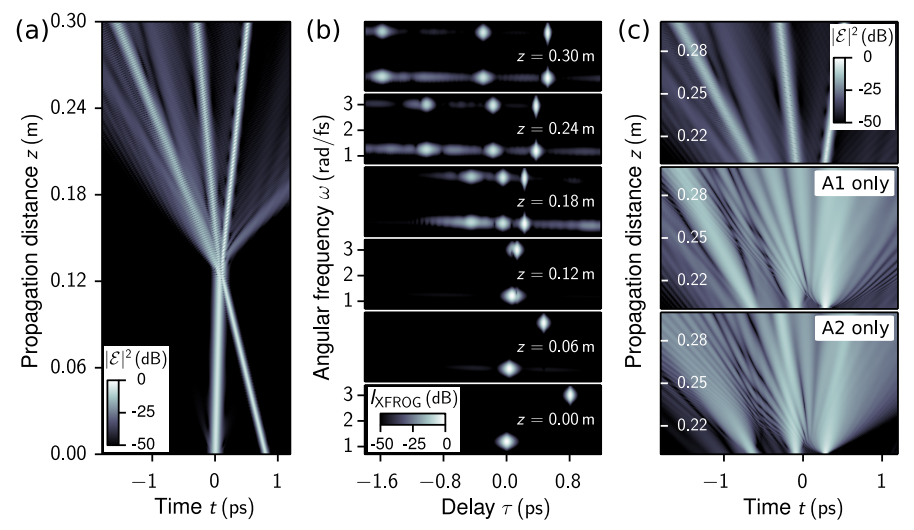

FIG. 4. Soliton molecule generation by collision. (a) Timedomain propagation of two colliding solitons leading to three molecule states and non-solitonic radiation (a movie MOV2 of the evolution is provided as supplemental material, see 42]). (b) Sequence of XFROG-traces at selected propagation distances (see supplemental material 42 for details). (c) Propagation of the bound states (top panel) and separate propagation of their isolated constituents in only A1 (middle panel) and A2 (bottom panel).

tual interaction $\mathrm{H}_{\text {int }}^{(12)}$ can be derived by filtering in the frequency domain (see supplemental material). As the energy of the bound state increases, the crossover from trapping to molecule formation is signaled by the mutual interaction dominating the self-interaction contribution to $\mathrm{H}_{\text {int }}$ (Fig. 3(e)), although the composite pulse loses a small amount of energy to free radiation (Fig. 3(d)).

In the next step we will address the more intriguing analogy to molecules related to a binding force. First we demonstrate how to induce molecule formation by soliton collision, in the spirit of formation of bound entities in a particle collider. Two solitons with temporal widths $t_{1 / 2}=25 / 15 \mathrm{fs}$ and frequencies $\omega_{1 / 2}=1.2 / 2.97 \mathrm{rad} / \mathrm{fs}$ are injected into the waveguide at time delay $\delta=800 \mathrm{fs}$ and energies, defined by their velocities. After the collision, we observe three localized states (Fig. 4(a)), propagating with different velocities. The collision process is accompanied by generation of strong non-solitonic radiation. The XFROG traces in Fig. 4 (b) provide deeper insight into the complex propagation dynamics. The three generated localized structures fulfil the criteria of compound states described above. Each of these states has a significant energy content in the two separated dispersion regimes, propagating in the time domain as one object. To demonstrate that these compounds only exist as an unit, we spectrally divide them into their separate parts in $A 1$ and $A 2$, and let these parts propagate independently from each other along the waveguide. As is evident from Fig. 4(c), the independent propagation of the isolated spectral components leads to nothing but linear dispersion of the pulses. This underlines again an analogy to a binding energy and especially the fact that this molecule state is not simply a co-propagation of two 
solitons.

In conclusion we presented the existence of previously unknown highly robust bound states of light providing a rich manifold of propagation dynamics and analogies to quantum mechanical molecules. In addition, we demonstrated that our optical system, which is fully classical, can mimic truly quantum-mechanical trapped states in an attractive potential well. This fact is of fundamental interest by itself. But the new states described here also hold promise for applications, e.g., communication systems as information can be decoded in the frequency domain, or for spectroscopy requiring coherent high-density spectra. In the presented study we focused on an exemplary photonic crystal fiber, but the scheme can easily be transferred to other fibers or waveguides, such as silicon slot waveguides or gas-filled Kagome fibers, allowing to address a wide range of pulse parameters. Moreover, the underlying main conditions, given by the quasi groupvelocity matched co-propagation of two solitons at vastly different frequencies, open up new perspectives for studying further novel phenomena, concerning soliton physics and analogies to other fields of physics.

The authors acknowledge financial support from Deutsche Forschungsgemeinschaft (DFG) (projects BA4156/4-2, MO 850-20/1), Germanys Excellence Strategy within the Cluster of Excellence PhoenixD (Photonics, Optics, and Engineering Innovation Across Disciplines) (EXC 2122, projectID 390833453), the Government of the Russian Federation (Grant 074-U01) through the ITMO University fellowship, and the European Union's Horizon 2020 research and innovation programme under the Marie Sklodowska-Curie grant agreement No 713694.

[1] P. G. Drazin and R. S. Johnson, Solitons: An Introduction, (Cambridge University Press, Cambridge, 1989).

[2] Y. S. Kivshar and G. P. Agrawal, Optical Solitons: From Fibers to Photonic Crystals, (Academic Press, San Diego, 2003).

[3] G. I. Stegeman and M. Segev, Science 286, 1518-1523 (1999).

[4] F. Mitschke, Fiber Optics: Physics and Technology, (Springer, Berlin, 2010).

[5] H. A. Haus and W. S. Wong, Rev. Mod. Phys. 68, 423444 (1996).

[6] C. Becker, S. Stellmer, P. Soltan-Panahi, S. Dörscher, M. Baumert, E. M. Richter, J. Kronjäger, K. Bongs, and K. Sengstock, Nat. Phys. 4, 496-501 (2008).

[7] J. Elgin, Nature (London) 313, 180-181 (1985).

[8] M. Stratmann, T. Pagel, and F. Mitschke, Phys. Rev. Lett. 95, 143902 (2005).

[9] N. J. Zabusky and M. D. Kruskal, Phys. Rev. Lett. 15, 240 (1965).

[10] M. N. Islam, C. D. Poole, and J. P. Gordon, Opt. Lett. 14, 1011-1013 (1989).

[11] A. Armaroli, C. Conti, and F. Biancalana, Optica 2, 497-
504 (2015).

[12] P. Rohrmann, A. Hause, and F. Mitschke, Sci. Rep. 2, 866 (2012).

[13] A. Hause, H. Hartwig, M. Böhm, F. Mitschke, Phys. Rev. A 78, 063817 (2008).

[14] A. V. Buryak and N. N. Akhmediev, Phys. Rev. E 51, 3572-3578 (1995).

[15] B. A. Malomed, Phys. Rev. E 47, 2874-2880 (1993); A. Zavyalov, R. Iliew, O. Egorov, and F. Lederer, Phys. Rev. A 80, 043829 (2009).

[16] I. Oreshnikov, R. Driben, and A. Yulin, Phys. Rev. A 96, 013809 (2017).

[17] N. N. Akhmediev, A. Ankiewicz, and J. M. Soto-Crespo, Phys. Rev. Lett. 79, 4047-4051 (1997).

M. Haelterman, S. Trillo, and P. Ferro, Opt. Lett. 22, 84-86 (1997).

[18] D. Neshev, E. Ostrovskaya, Y. Kivshar, and W. Krolikowski, Opt. Lett. 28, 710-712 (2003).

[19] V. V. Afanasjev and V. A. Vysloukh, J. Opt. Soc. Am. B 11, 2385-2393 (1994).

[20] E. N. Tsoy and F. Kh. Abdullaev, Phys. Rev. E 67, 056610 (2003).

[21] B. A. Umarov, N. A. B. Aklan, B. B. Baizakov, and F. Kh. Abdullaev, Opt. Phys. 49, 125307 (2015).

[22] Z. Chen, M. Acks, E. A. Ostrovskaya, and Y. S. Kivshar, Opt. Lett. 25, 417-419 (2000).

[23] Y. V. Kartashov, L. C. Crasovan, D. Mihalache, and L. Torner, Phys. Rev. Lett. 89, 273902 (2002).

[24] G. Xu, A. Gelash, A. Chabchoub, V. Zakharov, and B. Kibler, Phys. Rev. Lett. 122, 084101 (2019).

[25] X. Liu, X. Yao, and Y. Cui, Phys. Rev. Lett. 121, 023905 (2018).

[26] K. Krupa, K. Nithyanandan, U. Andral, P. TchofoDinda, and P. Grelu, Phys. Rev. Lett. 118, 243901 (2017).

[27] Z. Q. Wang, K. Nithyanandan, A. Coillet, P. TchofoDinda, and Ph. Grelu, Nature Communications 10, 830 (2019).

[28] A. Demircan, Sh. Amiranashvili, and G. Steinmeyer, Phys. Rev. Lett. 106, 163901 (2011).

[29] A. Demircan, Sh. Amiranashvili, C. Brée, and G. Steinmeyer, Phys. Rev. Lett. 110, 233901 (2013); A. Demircan, Sh. Amiranashvili, C. Brée, C. Mahnke, F. Mitschke, and G. Steinmeyer, Sci. Rep. 2, 850 (2012); D. V. Skryabin and A. V. Gorbach, Rev. Mod. Phys. 82, 1287 (2010).

[30] Sh. Amiranashvili and A. Demircan, Phys. Rev. A 82, 013812 (2010).

[31] V. N. Serkin, V. A. Vysloukh, and J. R. Taylor, Electron. Lett. 29, 12 (1993).

[32] E. N. Tsoy and C. M. de Sterke, Phys. Rev. A 76, 043804 (2007).

[33] A. Ferrando, E. Silvestre, J. J. Miret, and P. Andres, Opt. Lett. 25, 790 (2000).

[34] L. Zhang, Q. Lin, Y. Yue, Y. Yan, R. G. Beausoleil, and A. E. Willner, Opt. Express 20, 1685 (2012).

[35] S. Roy, and F. Biancalana, Phys. Rev. A 87, 025801 (2013).

[36] M. Zeisberger and M. A. Schmidt, Sci. Rep. 7, 11761 (2017); B. Debord, F. Amrani, L. Vincetti, F. Gérôme, and F. Benabid, Fibers 7, 16 (2019).

[37] R. Smith, Math. Proc. Camb. Phil. Soc. 78, 517 (1975).

[38] C. M. de Sterke, Opt. Lett. 17, 914 (1992).

[39] B. W. Plansinis, W. R. Donaldson, and G. P. Agrawal, 
Phys. Rev. Lett. 115, 183901 (2015).

[40] T. G. Philbin, C. Kuklewicz, S. Robertson, S. Hill, F. König, and U. Leonhardt, Science 319, 1367 (2008); D. Faccio, Cont. Phys. 1, 1 (2012).

[41] E. Feigenbaum and M. Orenstein, Opt. Exp. 12, 2193 (2004).

[42] See Supplemental Material at [URL] for technical details, analysis methods and supporting information including supplemental movies MOV1, MOV2, and Refs. [45-71].

[43] K. Tam, T. Alexander, A. Blanco-Redondo, and M. de Sterke, CLEO Europe EF-9.5 (to appear)(2019).

[44] A. V. Yulin, D. V. Skryabin, and P. St. J. Russell, Opt. Lett. 292411 (2004); D. V. Skryabin and A. V. Yulin, Phys. Rev. E 72, 016619 (2005); F. Biancalana, D. V. Skryabin and A. V. Yulin, Phys. Rev. E 70, 016615 (2004). 